\title{
ANÁLISE DA MORTALIDADE NA LISTA DE ESPERA DE FÍGADO NO PARANÁ, BRASIL. O QUE DEVEMOS FAZER PARA ENFRENTAR A ESCASSEZ DE ÓRGÃOS?
}

\author{
Analysis of liver waiting list mortality in Paraná, Brazi. What shall we do to face organ shortage?
}

Fábio SILVEIRA, Fábio Porto SILVEIRA, Matheus Martin MACRI, João Eduardo Leal NICOLUZZI

Trabalho realizado Instituto para Cuidado do Fígado (www.icfigado.org), Curitiba, PR, Brazil.

DESCRITORES - Transplante de fígado. Lista de espera. Mortalidade.
RESUMO - Racional - O transplante hepático é a melhor modalidade terapêutica para pacientes em estágio final de doença hepática. Minimização de morte, enquanto se espera o procedimento, envolve priorização de acordo com o estado clínico e a alocação adequada de fígados de doadores. Objetivo - Análise da mortalidade na lista de espera de fígado no estado do Paraná, PR, Brasil. Métodos - Foram analisados os dados sobre todos os pacientes $(n=65)$ que foram registrados na lista de espera de fígado durante um período de 32 meses. Resultados - A morte em lista de espera foi de $41,5 \%$ ( $n=27)$. Nenhuma diferença estatística foi observada em relação aos MELD / MELD-Na entre o grupo que faleceu $(19,88$ / 21,6) e não morreu $(17,28$ / 19,47). MELD-Na previu maior mortalidade, especialmente no subgrupo de pacientes com gravidade intermediária da doença (classe B) previsto pelo escore de CTP. Conclusão - É crítica a escassez de doadores de órgãos nessa região e a taxa de mortalidade em lista de espera excede em muito o risco inerente de um transplante de fígado, especialmente entre pacientes com MELD mais baixos. É desejável a utilização de um protocolo agressivo de doadores com critérios expandidos, split liver e transplante de doador vivo.

\begin{abstract}
Correspondência:
Fábio Silveira,

e-mail: silveira.fabio@gmail.com

Fonte de financiamento: não há

Conflito de interesses: conteúdo desse artigo é de responsabilidade dos autores e não necessariamente reflete a opinião ou a política do Sistema Nacional de Transplantes. O trabalho foi financiado pelo Instituto para Cuidado do Fígado. Potenciais conflitos de interesse não são relatados nesse artigo
\end{abstract}

Recebido para publicação:

Aceito para publicação:

HEADINGS - Liver transplantation. Waiting list. Mortality.
ABSTRACT - Background - Orthotopic liver transplantation is the best therapeutic modality for patients with end stage of liver disease. Minimization of death, while waiting for the procedure, involves accurate priorization according to clinical status and appropriate allocation of donor livers. Aim - The mortality analysis in the liver waiting list in Paraná state, PR, Brazil. Methods - Were analyzed the data on all patients $(n=65)$ who were registered on the liver waiting list during a 32 months period in the state of Paraná, southern Brazil. Results - The death rated in waiting list was $41,5 \%(n=27)$. No statistic difference was observed regarding the MELD/MELD-Na scores between the group who died $(19,88 / 21,6)$ and not died $(17,28 / 19,47)$. MELD-Na predicted a higher mortality, especially in the subgroup of patients with intermediate severity of disease (class B) predicted by the CTP score. Conclusion - It's critical the shortage of donor organs in our region, waiting list mortality rate far exceeds the inherent risk of a liver transplant, especially among patients with lower MELD scores. It's desirable to use an aggressive protocol of expanded criteria donors, split liver and living donor transplant.

\section{INTRODUÇÃO}

$\mathrm{O}$ transplante ortotópico de fígado (TOF) é uma modalidade terapêutica bem estabelecida para pacientes com doença hepática terminal. Avanços no manejo desses pacientes, assim como na imunossupressão e técnica cirúrgica têm contribuído para as significativas melhoras desde o primeiro TOF em 1963 (EUA) e 1968 (Brasil) ${ }^{11}$. A minimização da mortalidade em lista de espera de transplante hepático envolve precisa priorização de acordo com o estado clínico do paciente e 
apropriada alocação dos fígados doados. As ferramentas clínicas para determinação do prognóstico dos pacientes com doença hepática terminal, o escore de ChildTurcotte-Pugh (CTP) e o modelo prognóstico de doença hepática terminal (MELD) ${ }^{5}$, possuem limitações bem descritas ${ }^{16}$, não obstante são amplamente utilizados ${ }^{5,18}$. A alocação de fígados para transplante no Brasil, de doadores em morte encefálica, é baseada em critérios de urgência (MELD) desde 2006. A posição em lista pode ser modificada por situações específicas de urgência médica ou pontos de "bônus" para algumas doenças.

O potencial da substituição de um fígado doente está longe de ser realizado devido à discrepância entre o grande e crescente número de potenciais receptores e a insuficiente fonte de órgãos cadavéricos para transplante.

A resultante mortalidade em lista é o objeto de análise deste estudo.

\section{MÉTODOS}

O projeto deste estudo foi aprovado pela comissão de ética da instituição.

Os dados dos pacientes registrados na lista de espera de transplante de fígado do Instituto para Cuidado do Fígado, de janeiro de 2009 a agosto de 2011 foram coletados. Os dados estavam disponíveis por meio de protocolos eletrônicos que prospectivamente coletam os dados do pré-transplante, transplante e seguimento. Os dados de registro continham informações demográficas, clínicas e laboratoriais. A população em estudo incluiu todos os candidatos a transplante hepático ( $\geq 15$ anos) que estiveram na lista de espera em qualquer momento durante o período de estudo. O escore MELD, e sua modificação MELD-Na, foram calculados utilizando a fórmula-padrão ${ }^{8}$, assim como o escore Child-TurcottePugh ${ }^{5}$, ambos calculados na admissão do paciente em lista de espera. Pacientes foram considerados "óbitos em lista" quando no momento do óbito estavam ativos na lista ou se foram removidos por estarem em deterioradas condições clínicas para se submeterem ao procedimento. Em um esforço para minimizar bias de seleção, tratou-se esses pacientes como um endpoint combinado, pois ambos eventos representam falha de tratamento do sistema de alocação. Dados numéricos foram expressos como média \pm desvio-padrão. As análises estatísticas utilizaram testes de Kruskal-Wallis e consideraram valores de $p$ menores que 0.05 como significantes. As análises e gráficos de dados foram realizados utilizando o software EpiInfo ${ }^{\mathrm{TM}}$, versão 3.5.1 (Centro de Prevenção e Controle de Doenças).

\section{RESULTADOS}

Um total de 69 pacientes foram incluídos na lista de espera de transplante no período. Quatro foram excluídos por perda de seguimento, resultando em 65 pacientes. As características clínicas e os dados demográficos estão demonstrados na Tabela 1. A média de idade dos pacientes foi de 51.9 anos (variando de 19 a 67). Dos 65 pacientes, 12 eram do sexo feminino e 53 masculino. O índice de massa corpórea médio (IMC) foi 27.1 (variando de 20 a 36). Quarenta por cento dos pacientes possuíam o álcool como causa de sua doença e aproximadamente um terço possuía a cirrose induzida por infecção viral crônica. Doença maligna e insuficiência hepática aguda (IHA) foram responsáveis pela indicação de transplante em 10,8\% e $6,2 \%$, respectivamente. Outras causas representaram $13,7 \%$ dos casos.

Os 25 pacientes que morreram e os dois que

TABELA 1 - Características no momento de entrada em lista de espera

\begin{tabular}{|c|c|c|c|}
\hline & N / Média & $\begin{array}{c}\text { Desvio-padrão, } \\
\text { (limites), \% }\end{array}$ & $\begin{array}{c}95 \% \text { IC da } \\
\text { media }\end{array}$ \\
\hline Pacientes & 65 & & \\
\hline Idade (anos) & 51.9 & $9.67(19-67)$ & $46-58$ \\
\hline Masculino/feminino & $53 / 12$ & $81.5 / 18.5$ & \\
\hline IMC & 27.1 & 3.86 & $24.6-29.1$ \\
\hline \multicolumn{4}{|l|}{ Causa da doença } \\
\hline Cirrose - alcoólica & 26 & 40 & \\
\hline Cirrose - vírus & 19 & 29.3 & \\
\hline Malignidade & 7 & 10.8 & \\
\hline IHA & 4 & 6.2 & \\
\hline Outros & 9 & 13.7 & \\
\hline MELD escore & 18.36 & $7.25(8-38)$ & $13-20$ \\
\hline MELD-Na escore & 20.36 & $7.08(8-38)$ & $16-23$ \\
\hline CTP escore A & 10 & 15.4 & \\
\hline CTP escore B & 30 & 46.2 & \\
\hline CTP escore C & 25 & 38.5 & \\
\hline
\end{tabular}

foram removidos por piora da condição clínica foram considerados como um único grupo, resultando em taxa de mortalidade em lista de espera de $41,5 \%$. A análise univariada comparando os pacientes que morreram e não morreram em lista de espera, considerando a idade, sexo, IMC, distância da cidade de origem ao centro de transplante, o MELD escore médio (19.88 vs 17.28), o MELD-Na escore médio (21.62 vs 19.47), respectivamente, não apresentou diferenças estatisticamente significantes. O tempo médio de espera em lista foi de 70 dias para os pacientes que morreram e 169 dias para os que não morreram, diferença estatisticamente significante entre os grupos (Tabela 2).

A comparação do número de dias em lista de espera, por tipo sanguíneo, resulta no gráfico demonstrado na Figura 1.

A análise da mortalidade em 90 dias prevista pelo escore MELD e MELD-Na, estratificada por grupos demonstrou diferença estatisticamente significante (Tabela 3).

A média dos escores MELD e MELD-Na entre os pacientes transplantados e não transplantados, sendo neste incluídos os pacientes que morreram e os que ainda estão em lista de espera, estão demonstrados na Tabela 4, não apresentando diferença estatística significante. 
TABELA 2 - Estratificação dos grupos

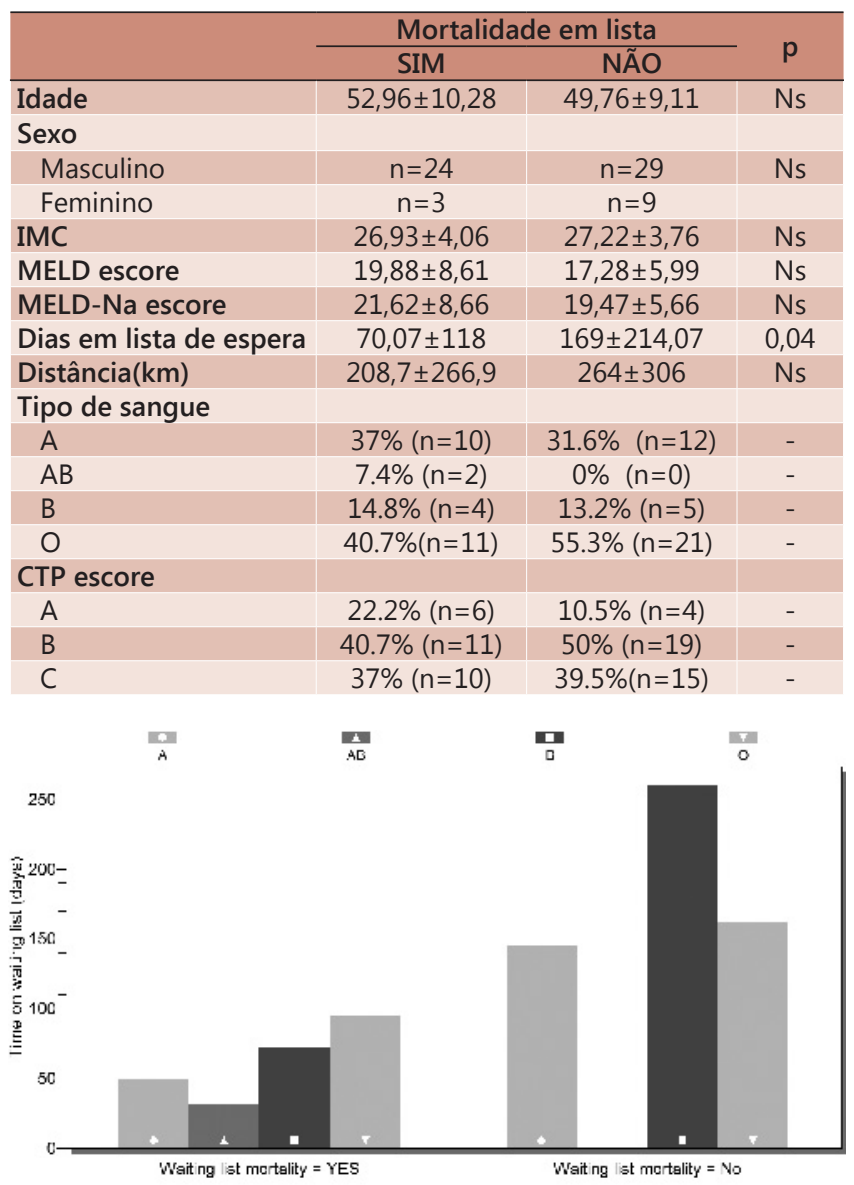

FIGURA 1 - Número de dias em lista de espera estratificado pelo tipo sanguíneo

TABELA 3 - Mortalidade prevista em 90 dias entre os grupos

\begin{tabular}{|lccc|}
\hline & \multicolumn{2}{c}{ Mortalidade em lista } & \multirow{2}{*}{$p$} \\
\cline { 2 - 3 } & \multicolumn{1}{c}{ SIM } & NÃO & \\
\hline MELD escore & $0.29 \pm 0.26$ & $0,20 \pm 0,17$ & Ns \\
\hline MELD-Na escore & $0.34 \pm 0.26$ & $0,25 \pm 0,17$ & Ns \\
\hline$p$ & 0.0001 & 0.0211 & \\
\hline
\end{tabular}

Valores expressos pela média $\pm \mathrm{DP}$

TABELA 4 - Diferenciação entre o grupo transplantado e não-transplantado

\begin{tabular}{lccc}
\hline & \multicolumn{2}{c}{ Transplante } & \multirow{2}{*}{$p$} \\
\cline { 2 - 3 } & \multicolumn{1}{c}{ SIM } & NÃO & \\
\hline MELD escore & $19.2 \pm 6.78$ & $17.93 \pm 6.78$ & Ns \\
\hline MELD-Na escore & $21.09 \pm 6.25$ & $20 \pm 7.51$ & Ns
\end{tabular}

Valores expressos pela média $\pm \mathrm{DP}$

TABELA 5 - Comparação do MELD e MELD-NA: pacientes estratificados pelo escore CTP

\begin{tabular}{lccc}
\hline & MELD & MELD-Na & P \\
\hline CTP escore A & $12.2 \pm 3.3$ & $13.3 \pm 3.5$ & Ns \\
\hline CTP escore B & $17 \pm 5.6$ & $19.4 \pm 5.6$ & 0.0248 \\
\hline CTP escore C & $22.4 \pm 7.8$ & $24.2 \pm 7.3$ & Ns
\end{tabular}

Valores expressos pela média $\pm \mathrm{DP}$
A comparação entre o MELD e MELD-Na estratificado de acordo com o escore CTP, demonstra uma diferença significante no grupo B do CTP, demonstrada na Tabela 5.

Estratificando-se três grupos de escores MELD, designados pacientes de baixo (MELD 10-15), médio (MELD 16-20) e alto-risco (MELD > 21), pode-se observar a distribuição entre os que morreram, os transplantados e os que estão esperando o transplante, assim como a mortalidade em 90 dias prevista pelo MELD.

\section{DISCUSSÃO}

O transplante hepático possui um grande impacto no cuidado dos pacientes com doença hepática terminal, sendo que seu maior objetivo é a minimização da mortalidade em lista. Os esforços para a melhoria dos critérios de alocação de órgãos para TOF em virtude da escassez de órgãos são contínuos e sempre matéria de debates.

Nesta coorte observaram-se taxas de mortalidade mais altas do que as previstas pelos escores MELD e MELD-Na. O escore MELD é eficaz na previsão de mortalidade em três meses de pacientes com doença hepática terminal|4,8,13,14,15, entretanto, seu uso demonstrou algumas de suas limitações, como a dificuldade em prever a mortalidade de pacientes com ascite refratária, hiponatremia, história de hemorragia digestiva alta e encefalopatia hepática $a^{6,8,9}$.

Demonstra-se que o escore MELD-Na previu maior mortalidade no subgrupo de pacientes com gravidade média (classe B) de doença hepática prevista pelo escore de CTP. Esse achado corrobora com o fato que o escore MELD-Na é considerado mais eficaz na previsão de mortalidade ${ }^{9}$, pelo fato de que a hiponatremia tem sido associada à síndrome hepatorrenal, ascite e morte por doença hepática ${ }^{1,3}$. Essa diferença é ainda mais pronunciada entre os pacientes com escore MELD baixo ${ }^{2,8}$, diferença essa observada também neste estudo.

O escore de CTP é útil como um meio rápido de prever o risco relativo de mortalidade entre grupos de pacientes com cirrose, sendo amplamente utilizado na estratificação de pacientes para transplante em virtude de sua simplicidade e facilidade de uso ${ }^{16}$. Seu uso é limitado pelo fato de que as variáveis ascite e encefalopatia são subjetivas e influenciadas pela terapêutica médica, assim como pela falta de avaliação da função renal, notadamente conhecida como importante fator prognóstico na cirrose ${ }^{6}$.

Este estudo demonstra evidência que é crítica a falta de órgãos para transplante em nossa região. 0 sistema brasileiro de alocação de órgãos é baseado na gravidade da doença e regionalizado pela localização da residência do receptor. Atualmente aqui a taxa efetiva de doação é de 9.2 pmp (por milhão de população), enquanto em outras unidades da federação essas taxas atingem 20-25 pmp. 
Métodos para aumentar o número de transplantes incluem o uso de doadores com critérios expandidos ${ }^{7}$, fígado dividido ${ }^{11}$ e doadores vivos ${ }^{17}$, apesar da segurança do doador, neste último caso, permanecer como uma preocupação constante ${ }^{15}$. A mortalidade nesta lista excede em muito os riscos inerentes de um procedimento de transplante hepático, especialmente em pacientes com MELD escore mais baixo.

\section{CONCLUSION}

É necessário utilizar-se uma combinação dos escores de MELD, MELD-NA e CTP, assim como outros sinais cardinais de gravidade da doença, como hemorragia digestiva alta e encefalopatia hepática na estratificação de risco de nossos pacientes. O subgrupo de maior mortalidade, conforme evidenciado por esses dados, deve, portanto, ser manejado com um protocolo agressivo de utilização de doadores com critérios expandidos, fígado dividido e transplante intervivos.

\section{REFERÊNCIAS}

1. Arroyo V, Colmenero J. Ascites and hepatorenal syndrome in cirrhosis: pathophysiological basis of therapy and current management. J Hepatol 2003;38 Suppl 1:S69-89.

2. Biggins SW, Kim WR, Terrault NA, et al. Evidence-based incorporation of serum sodium concentration into MELD. Gastroenterology 2006;130(6):1652-60.

3. Borroni G, Maggi A, Sangiovanni A, Cazzaniga M, Salerno F. Clinical relevance of hyponatraemia for the hospital outcome of cirrhotic patients. Dig Liver Dis 2000;32(7):605-10.

4. Dunn W, Jamil LH, Brown LS, et al. MELD accurately predicts mortality in patients with alcoholic hepatitis. Hepatology 2005;41(2):353-8.

5. Durand F, Valla D. Assessment of the prognosis of cirrhosis: ChildPugh versus MELD. J Hepatol 2005;42 Suppl(1):S100-7.
6. Freitas ACT, Coelho JCU, Parolin MB, Marias JEF, Zeni-Neto C, Gonçalves CG. Fatores de risco e conduta nas complicações do trato biliar no transplante hepático. Rev. Col. Bras. Cir. 2000; 27(6):359-365.

7. Gastaca M. Extended criteria donors in liver transplantation: adapting donor quality and recipient. Transplant Proc 2009;41(3):975-9.

8. Heuman DM, Abou-Assi SG, Habib A, et al. Persistent ascites and low serum sodium identify patients with cirrhosis and low MELD scores who are at high risk for early death. Hepatology 2004;40(4):802-10.

9. Kim WR, Biggins SW, Kremers WK, et al. Hyponatremia and mortality among patients on the liver-transplant waiting list. $\mathrm{N}$ Engl J Med 2008;359(10):1018-26.

10. Kremers WK, van IM, Kim WR, et al. MELD score as a predictor of pretransplant and posttransplant survival in OPTN/UNOS status 1 patients. Hepatology 2004;39(3):764-9.

11. Murray KF, Carithers RL, Jr. AASLD practice guidelines: Evaluation of the patient for liver transplantation. Hepatology 2005;41(6):140732.

12. Renz JF, Emond JC, Yersiz H, Ascher NL, Busuttil RW. Split-liver transplantation in the United States: outcomes of a national survey. Ann Surg 2004;239(2):172-81.

13. Rosen HR, Prieto $M$, Casanovas-Taltavull $T$, et al. Validation and refinement of survival models for liver retransplantation. Hepatology 2003;38(2):460-9.

14. Said A, Williams J, Holden J, et al. Model for end stage liver disease score predicts mortality across a broad spectrum of liver disease. J Hepatol 2004;40(6):897-903.

15. Silva OC, Souza FF, Nejo P. Doação de órgãos para transplantes no Brasil: o que está faltando? O que pode ser feito? ABCD Arq Bras Cir Dig. 2011;24(2):93-94

16. Surman OS. The ethics of partial-liver donation. N Engl J Med 2002;346(14):1038.

17. Trotter JF, Brimhall B, Arjal R, Phillips C. Specific laboratory methodologies achieve higher model for endstage liver disease (MELD) scores for patients listed for liver transplantation. Liver Transpl 2004;10(8):995-1000.

18. Trotter JF, Wachs M, Everson GT, Kam I. Adult-to-adult transplantation of the right hepatic lobe from a living donor. $\mathrm{N}$ Engl J Med 2002;346(14):1074-82.

19. Wiesner RH, McDiarmid SV, Kamath PS, et al. MELD and PELD: application of survival models to liver allocation. Liver Transpl 2001;7(7):567-80. 